\title{
Resíduos sólidos: evidências científicas sobre sua disposição final e impactos ambientais
}

A Gestão dos Resíduos Sólidos Urbanos (RSU) é uma das grandes demandas ambientais do mundo contemporâneo e os problemas enfrentados por sua destinação inadequada são ainda maiores por causa do aumento desproporcional da geração de resíduos em volume, resultante de um contexto de maior urbanização, crescimento populacional e globalização econômica. Tendo em vista que atualmente as principais formas de destinação dos Resíduos Sólidos utilizadas não tem conseguido solucionar de maneira satisfatória a maioria dos problemas ambientais, existe cada vez mais a necessidade de encontrar não só alternativas para disposição final, mas opções que considerem o problema de maneira sistêmica evidenciando modelo produtivo e ambiental como um todo a fim de formular estratégias, promover a sustentabilidade ambiental e a melhoria da qualidade de vida da população. Nesse contexto, a presente pesquisa foi realizada objetivandose analisar as contribuições mais recentes publicadas na literatura científica, através da revisão sistêmica, pela consulta de bancos de dados virtuais de relevância mundial a fim de avaliar a eficiência ambiental e econômica partindo como princípio a disposição final dos RSU em diferentes países. Diante dos resultados obtidos verifica-se o crescimento acelerado das pesquisas que tratam dos impactos ambientais associados a disposição de resíduos, sendo evidenciados em maior proporção as áreas afetadas em detrimento dos impactos provocados aos seres vivos.

\section{Solid waste: scientific evidence on its final disposal and environmental impacts}

\begin{abstract}
The management of municipal solid waste (MSW) is one of the great environmental demands of the contemporary world and the problems faced by your improper disposal are even greater because of the disproportionate increase of waste generation in volume resulting from a context of greater urbanization, population growth and economic globalization. Since currently the main forms of solid waste disposal used hasn't been able to resolve satisfactorily the most environmental problems, there is more and more the need to find alternative not only for final disposition, but options that consider the issue systemically highlighting environmental and productive model to formulate strategies, promote environmental sustainability and improving the quality of life of the population. In this context, this research was carried out to analyze the most recent contributions published in the scientific literature, through the systemic review, by the query virtual database of world relevance in order to assess the environmental and economic efficiency starting as principle to final disposal of MSW in different countries. Before the results of the accelerated growth of the research dealing with the environmental impacts associated with waste disposal, being evidenced in greater proportion to the affected areas to the detriment of impacts to alive.
\end{abstract}

Keywords: Evaluation Methods; Systematic review of literature; Municipal Solid Waste.

Topic: Desenvolvimento, Sustentabilidade e Meio Ambiente

Reviewed anonymously in the process of blind peer
Received: $10 / 08 / 2018$

Approved: 24/09/2018
Dihego Sousa Pessoa (iD)

Universidade Federal de Campina Grande, Brasi

http://lattes.cnpq.br/8797044499355611

http://orcid.org/0000-0001-6954-4610

dihegopessoaa@hotmail.com

\section{Ana Maria Ferreira Cosme (id)}

Universidade Federal de Campina Grande, Brasil

http://lattes.cnpq.br/6562223986723856

http://orcid.org/0000-0002-1342-1950

cosmeferreira@hotmail.com

\section{Viviane Farias Silva (iD}

Universidade Federal de Campina Grande, Brasil

http://lattes.cnpq.br/5011520274887172

http://orcid.org/0000-0002-5891-0328

flordeformosur@hotmail.com

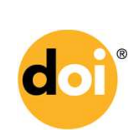

DOI: 10.6008/CBPC2179-6858.2018.007.0018

\author{
Rubenia de Oliveira Costa \\ Universidade Federal de Campina Grande, Brasil \\ http://lattes.cnpq.br/0790230556773806
}

rubeniacosta@yahoo.com.br

Vera Lucia Antunes de Lima (iD)

Universidade Federal de Campina Grande, Brasil

http://lattes.cnpq.br/5379077061489077

http://orcid.org/0000-0001-7495-6935

antunes.lima@hotmail.com

Aline Costa Ferreira

Universidade Federal de Campina Grande, Brasi

http://lattes.cnpq.br/3332107317093873

alinecosta@yahoo.com.br
Referencing this:

PESSOA, D. S.; COSME, A. M. F.; SILVA, V. F.; COSTA, R. O.; LIMA, V. L. A.; FERREIRA, A. C.. Resíduos sólidos: evidências científicas sobre sua disposição final e impactos ambientais. Revista Ibero Americana de Ciências Ambientais, v.9, n.7, p.202-210, 2018. DOI: http://doi.org/10.6008/CBPC2179-6858.2018.007.0018 


\section{INTRODUÇÃO}

O aumento da geração de resíduos sólidos urbanos (RSU) é uma preocupação que está no cotidiano da população de todo o mundo (PATEL et al., 2010), principalmente devido a necessidade do uso de estratégias específicas das gestões municipais. A crescente urbanização tem sido observada nas últimas três décadas (WANG et al., 2016), e tem provocado um significativo aumento na geração de resíduos, principalmente pelo elevado nivel de consumo das pessoas que resulta em descarte em menor período. Nos comerciais de televisão é estimulado a compra de produtos por meio de propagandas que incentiva sempre o consumo, mesmo que não seja necessário, para que assim haja movimento comercial acarretando em maior produção de residuos.

A produção de resíduos vem aumentando gradativamente e a destinação final deve ser planejada de maneira que evite a degradação ambiental e social. Para analisar a perspectiva dos residuos sólidos urbanos, conforme Jacobi (2012) relata a importância de levar em consideração as questões espaciais, ambientais, socioculturais, saúde e entre outros.

A disposição inadequada dos residuos sólidos urbanos diretamente no solo ocasiona poluição do solo, água e serve de ambiente para animais e pessoas, além de local atrativo para urubus tornando-se um problema para a saúde do animal e para a população que reside nas proximidades. Segundo Waldman (2010) os locais de despejo descontrolados dos RSU é o resultado da negligência dos poderes publicos com suas áreas mais pobres.

Algumas pessoas que trabalham com material reciclavel consideram estes locais de disposição final dos residuos como ambientes de trabalhos onde realizam a separação dos materiais recicláveis, estando vulnerável a diversas doenças, Alves et al. (2018) afirmam que ficar exposto por longo período nestas regiões insalubres de disposição inregular de RSU pode gerar variados problemas de saúde físisca e mental, além de disturbios ao meio ambiente. Sánchez et al. (2017) descrevem os riscos no trabalho no setor de resíduos sólidos, constatando acidentes de trabalho como cortes, excesso de cargas e acidentes ocasionados por queda de materiais, indicando aos trabalhadores que realizem a gestão de prevenção dos riscos que afetam diretamente a saúde e segurança.

A disposição dos residuos produzidos é comumente realizados em locais distantes das áreas urbanas, contudo com a crescente demanda de residuos e a expansão urbana, as residências ficaram proximas aos lixões, sendo incomondo e gerando transtornos bem como a degradação ambiental e social, sendo o fator impulsionante para que fosse estabelecido uma lei de Politica Nacional dos Residuos Sólidos (PNRS 12.305/2010) no Brasil para que as cidades se adequassem a disposição adequada dos residuos sólidos gerados. Lima et al. (2018) salientam que a criação da PNRS tem o intuito de limitar e administrar todos os tipos de residuos sólidos incluindo os considerados perigosos, atribuindo a obrigação à quem produz os residuos, a instituição governamental e a população.

Os aterros sanitários quando bem dimensionado e estruturado com impermeabilização não ocasionará impactos negativos, contudo o manejo inadequado implica em diversos problemas abrangentes, 
sendo o aterro uma das fontes mais recorrentes de contaminação das águas subterrâneas em todo o mundo. A fim de limitar seus impactos sobre os recursos hídricos subterrâneos, as atuais regulamentações ambientais impõem a adoção de medidas adequadas para a proteção da qualidade das águas subterrâneas (STEFANIA et al., 2018).

A gestão dos resíduos sólidos é importante para a recuperação de recursos, sustentabilidade e saúde, quando o descarte em aterros sanitários é realizado com capacidade mínima de reciclagem o esgotamento da capacidade de retenção dos aterros ocorre antes do tempo previsto segundo Khalil et al. (2018), sendo fundamental a reciclagem, aumentando o tempo útil dos aterros sanitários. $\mathrm{O}$ odor proveniente dos aterros sanitários, incomoda quem trabalha no local, assim como para comunidades próximas (LIU et a., 2018). Desse modo, nota-se a importância de utilizar formas de disposição final dos residuos sólidos adequada, bem como o manejo aplicado. Neste sentido, o presente artigo foi realizado objetivando-se analisar como a temática da Gestão Sustentável dos Resíduos Sólidos Urbanos está sendo abordada em pesquisas cientificas em relação a sua disposição final e impactos ambientais.

\section{MATERIAIS E MÉTODOS}

O método utilizado nesta pesquisa foi revisão sistêmica, segundo Galvão et al. (2014) a revisão sistemática é classificada como pesquisa secundária, porque seus aspectos são fundamentados em pesquisas primária como banco de dados. Sampaio et al. (2007) relatam que estas metodologias de pesquisa são metódicas, explicitas e passíveis de reprodução, tornando se importante diante dos progressivos dados científicos, abreviando as evidências cientificas disponíveis na literatura.

Galvão et al. (2014) afirmam que a revisão sistemática tem como objetivo identificar, selecionar, avaliar e sintetizar as informações importantes acessíveis, em relação a informações divergentes sobre algumas pesquisas, essa é uma das melhores formas de tornar compreensível as controvérsias cientificas. Os dados da pesquisa foram obtidos através da seleção de artigos científicos disponibilizados na rede mundial virtual, o software EndNote X8.2. A pesquisa foi realizada nos seguintes bancos de dados: PUB MED (NLM), Web of Science Collection, Web of Science (SCI) e Web of Science (SSCI).

Dantas Neto et al. (2016) relatam que este software possui banco de dados gratuito e interdisciplinar, com diversos periódicos nacionais e internacionais. O que possibilita abrangência no resultado da pesquisa a nível mundial sobre pesquisas cientificas em relação a temática abordada. Os termos em inglês utilizados para a busca foram: dumpsites, solid waste, degradation dumpsites, recovered landfills, landifills. Os artigos científicos que possuíam estes termos no title, sendo este o filtro utilizado no software.

Para cada termo foi verificado os artigos publicados do ano de 2000 até 2018, analisando a quantidade de artigos pôr termo e ano, averiguando o avanço das pesquisas cientificas em relação a esta temática. Apenas os artigos publicados nos meses de janeiro a maio do ano de 2018 para o termo dumpsites e landfills foram verificados os periódicos que foram publicados e avaliados os estudos referentes aos impactos gerados pela disposição dos resíduos sólidos. 


\section{RESULTADOS E DISCUSSÃO}

No banco de dados foram obtidos a evolução crescente das pesquisas cientificas, com os termos landfills (Figura 1A), solid waste (Figura 1B) e dumpsites (Figura 1C), porém para recovered landfills, degradation dumpsites, não houve nenhum registro. Para o termo landfills, observa-se na Figura $1 \mathrm{~A}$ uma tendência de interesse sobre esta temática, com maior registro no ano de 2007, em 2017 para termo solid waste (Figura 1B) devido o aumento da produção de resíduos sólidos e preocupação com sua destinação final. Na Figura 1C, percebe-se que o termo dumpsites há menores quantidades de artigos científicos em relação a este assunto, sendo que os lixões são os depósitos finais de resíduos sólidos sem nenhum tratamento prévio, degradando o meio ambiente e deixando a população disposta a diversos problemas de saúde.
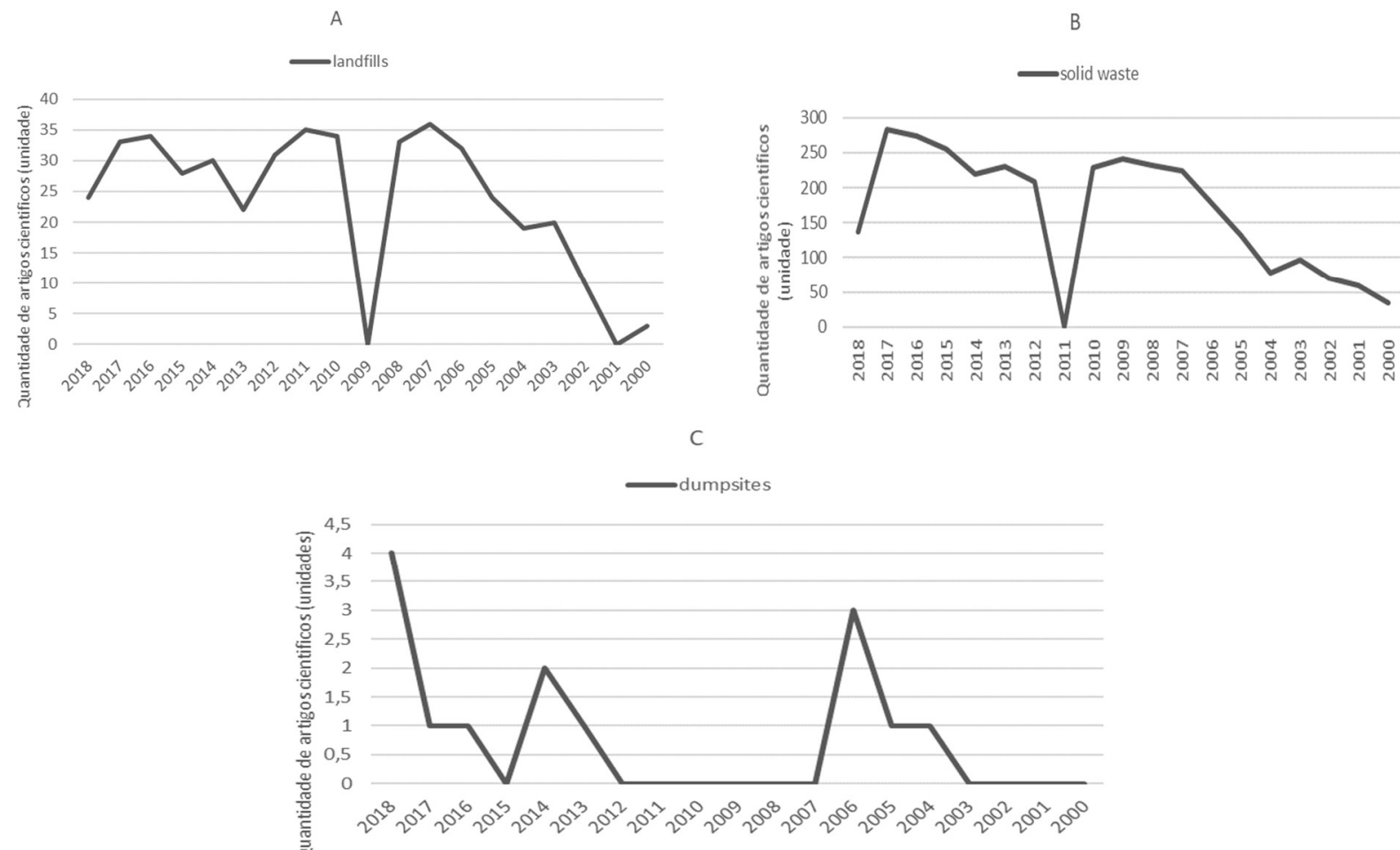

Figura 1: Quantidade de artigos publicados em periodicos num periodo de 2000 a 2018.

Al-Khatib et al. (2015) avaliando a percepção ambiental da população na Cisjordânia, averiguaram que $65 \%$ das pessoas são consciente dos impactos ambientais negativos em relação a disposição inadequada dos residuos sólidos, enquanto $41,6 \%$ sofrem com os lixões. Estes autores afirmam que que um dos principais impactos ambientais é a poluição do ar.

Pesquisando sobre pessoas que habitam em proximidades a aterro sanitário, Al-Delaimy et al. (2014) constataram que os moradores possuem maiores problemas de saúde, como cansaço, dermatológicos, problemas de concentração, entre outros, quando comparado com pessoas de outros locais. Akortia et al. (2017) estudando os metais pesados em área de disposição de resíduos eletrônicos, relataram evidências de ferro, cobre, chumbo e manganês, com maiores quantidades para ferro e cobre, constatando degradação do solo por poluição dos metais, sendo preocupante o cobre e chumbo para a população principalmente as crianças. Alterações em solos próximos a aterros industriais da Nigéria foram observadas por Ademola et al. 
(2014) com valores elevados para cobre, cadmio e chumbo, dessa maneira não é aconselhável a pratica da agricultura nessas áreas, por haver presença de metais pesados, sendo considerada uma área degradada.

No Zimbábue as disposições dos resíduos sólidos geralmente são utilizadas os lixões, local onde são despejados resíduos do município, industrias, estabelecimentos comerciais e de serviços socias. A população está exposta a enorme quantidade de poluentes via ar, água e comida, contaminados pelos lixiviados, como consequência da globalização o compartilhamento de alimentos contaminados e ônus relacionados a doenças (TONGESAYI et al., 2018). Para o termo dumpsites foram observados 4 registros no ano de 2018 e para landfills foram 24, verificando os periódicos em que foram publicados os artigos, Figura 2, há índice elevado para a Vaste Manage Res com o termo landfills até mês de maio do ano de 2018.

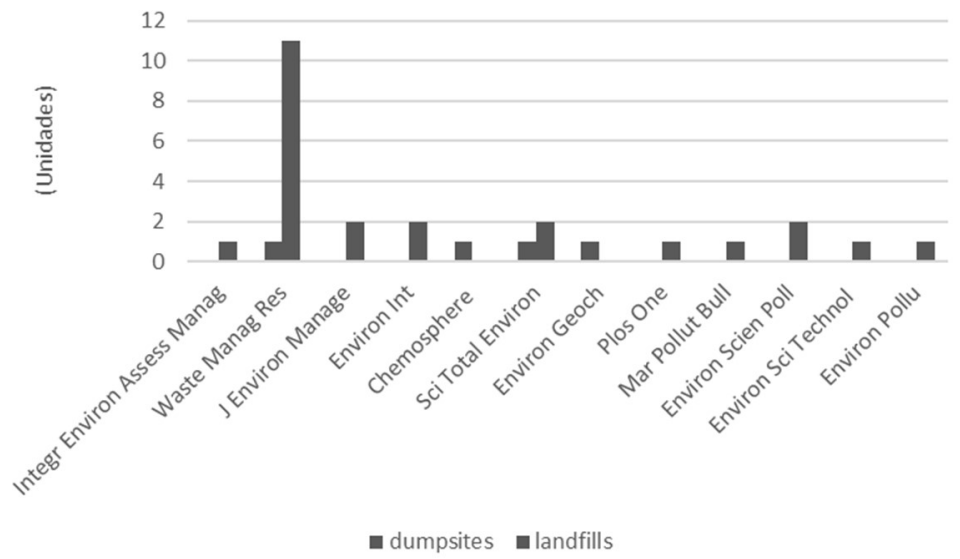

Figura 2: Quantidade de artigos publicados nos periodicos nos meses de janeiro a maio do ano de 2018.

Chakraborty et al. (2018) relatam a presença de compostos halogenados nos resíduos eletrônicos resultam em poluentes orgânicos persistentes, e perceberam que em áreas de lixões eletrônicos e lixões de forma em geral há crescente presença destas substâncias, com maiores valores de equivalentes de toxicidade máxima (TEQ) para dibenzo-p-dioxinas policloradas e dibenzofuranos (PCCD/Fs) no lixão Kodangaiyur de Chennai, Sul da Índia. Estes autores afirmam que a origem dos policlorobifenilos (PCB's) são produto da combustão de plásticos no fluxo de lixo eletrônico e resíduos despejados.

Os lixões são considerados uma das fontes de liberação dos gases do efeito estufa, como afirmam Ngwabie et al. (2018). Pesquisando sobre a emissão de metano $\left(\mathrm{CH}_{4}\right)$, óxido nitroso $\left(\mathrm{N}_{2} \mathrm{O}\right)$ e Dióxido de Carbono $\left(\mathrm{CO}_{2}\right)$ em dois locais de disposição inadequada de resíduos, estes autores constataram elevados índices para estes três gases em ambos os lixões, assim como verificaram variações nas emissões que pode ser justificado pela natureza heterogênea dos resíduos, estágios de decomposição e diferentes condições ambientais dentro dos resíduos, considerado uma fonte de poluição do ar que afeta a população e a atmosfera. Tongesayi et al. (2018) analisaram niveis de chumbo em dois locais de resíduos no Zimbábue, os níveis de $\mathrm{Pb}$ variaram de 23.000 a $14.600 .000 \mathrm{mcg} / \mathrm{Kg}$, com quantidade de exposição diária foram superiores a ingestão diária tolerável pela Organização Mundial de Saúde.

O encerramento de lixões no Líbano no ano de 2016, direcionou os resíduos municipais em ruas e florestas, sendo detectado que o principal problema dos resíduos sólidos dispostos inadequadamente iam direto para o Mar Mediterrâneo, Khalil et al. (2018), fizeram coleta de amostras de chorume destas áreas e 
verificaram que os danos nas enzimas hepática, baço e medula óssea dos animais, ficando evidente a necessidade de tratamento dos resíduos sólidos adequados. O'Shea et al. (2018) asseguram que antes das regulamentações ambientais, os aterros em ambientes eram freqüentemente construídos sem controle de lixiviados, contando com a atenuação natural nos sedimentos para diluir e dispersar os contaminantes, reduzindo o impacto ambiental, sendo ambientes com risco de poluição.

Os impactos ambientais e a saúde ocasionados pela disposição inadequada dos resíduos sólidos são inúmeros, foram observados nos artigos publicados no ano de 2018, Figura 3, os impactos ambientais ocasionados são mais evidentes nos aterros (landfills) e lixões (dumpsites), com maior evidencia de preocupações com a saúde para o uso do termo landfills. Alguns artigos utilizam para lixões os aterros abertos.

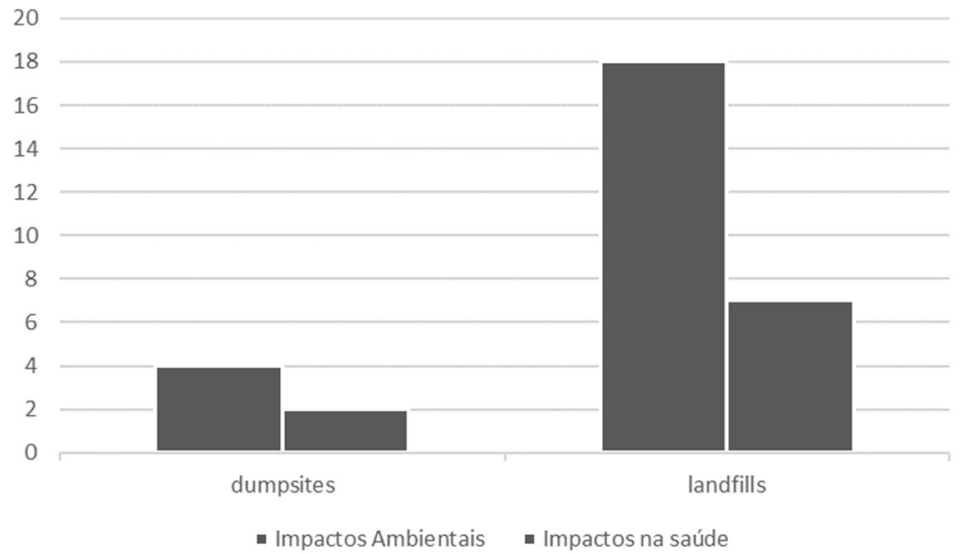

Figura 3: Artigos que objetivam em sua pesquisa os impactos ambientais e na saúde, no ano de 2018.

Liu et al. (2018) estudando as taxas de emissão de metilmercaptano $\left(\mathrm{CH}_{3} \mathrm{SH}\right)$, o sulfeto de dimetila, o dissulfeto de dimetila (DMDS) e o dissulfeto de carbono $\left(\mathrm{CS}_{2}\right)$, obtiveram para o DMDS a maior taxa de emissão $\left(7,18\right.$ mugm $\left.^{-2}\right)$ s $\left(^{-1}\right)$ e o $\mathrm{CH}_{3} \mathrm{SH}$ foi o composto odorífero dominante com uma taxa de emissão média de 4,58mugm $\left({ }^{-2}\right)$ s $\left({ }^{-1}\right)$. Configurando-se assim que não basta ter os residuos dispostos em aterros sanitarios, estes devem ter mecanismos que captem os gases para reduzir ao maximo os impactos ao meio ambiente e a população.

Os efeitos e mecanismos da biorremediação do solo contaminado com petróleo utilizando resíduos velhos (AR) de aterros sanitários, afirmam Liu et al. (2018) que o AR é um condicionador eficaz do solo e bioestimulador que pode melhorar a qualidade do solo contaminado com petróleo e promover o crescimento microbiano, sendo um método econômico e fácil de usar que facilita a aplicação in situ em larga escala, ao mesmo tempo em que recicla grandes quantidades de AR dos aterros sanitários.

O aumento na população humana das cidades levou a um aumento na quantidade de resíduos depositados em aterros sanitários, instalações que se tornaram um novo recurso alimentar para espécies ameaçadas e de pragas, tais como abutres,Tauler-Ametller et al.(2018) relatam que $50 \%$ de casais reprodutores alimentam-se de alimentos oriundos de lixões e a redução destes animais em todo mundo está diretamente relacionado ao consumo e disponibilidade de recursos alimentares. 
Avaliando as emissões de metano em 91 aterros na Dinamarca em relação a redução da emissão deste gás com a construção de capas biologicas, Fredenslund et al. (2018) averiguaram que estas reduções foram significantes em 25 aterros foram considerados relevantes quando o limite de emissão de metano quando considerado $2 \mathrm{~kg} \mathrm{CH} 4 \mathrm{~h}^{-1}$. O'Shea et al. (2018) trabalhando com contaminação por metais em sedimentos de sal marinho em nações industrializadas, obtiveram uma carga de metal baixa de 1200 e 1650 kg Pb e Zn, apesar de mais de 1000 aterros históricos têm risco de inundação ou erosão das costas no Reino Unido, considerando estas áreas fontes de poluição difusa significativa.

Os aterros sanitários são reservatórios de antibióticos, metais pesados, desinfetantes e outros contaminantes emergentes, e estão intimamente associados à crescente prevalência de genes de resistência a antibióticos (YOU et al., 2018). Foram selecionados sete antibioticos (cefalexina [CLX], cloranfenicol [CAP], ciprofloxacina [CIP], eritromicina [ERY], roxitromicina [ROX], trimetoprim [TMP], sulfametoxazol [SMX]) em um dos maiores aterros ativos na Ásia e dois aterros que foram fechados por 20 anos para examinar ocorrências de antibióticos em lixiviados, Chung et al. (2018) observaram que o CLX, CAP, ROX e SMX em lixiviados não excederam as concentrações de efeito não esperado de resistência a antibióticos (ABR) (PNECs), mas houve excedências para CIP, ERY e TMP em alguns locais de estudo e em algumas datas, concluiram que deve se ter entendimento avançado das práticas de acesso, uso e descarte de produtos farmacêuticos, eficácia de estratégias de intervenção (por exemplo, tecnologias de tratamento de lixiviados, esquemas de recuperação de medicamentos) e contribuições de lixiviados para o ciclo de vida de antibióticos e ABR. desenvolvimento, particularmente em regiões costeiras de rápida urbanização, com sistemas de gestão de resíduos menos avançados do que Hong Kong.

Tian et al. (2018) verificaram a ocorrência e distribuição de substancias polifluoralquílicas (PFASs) no ar, deposição a seco e nas folhas de plantas de dois aterros em Tianjin/China, as concentrações máximas de PFAS nos dois aterros foram de até 9,5 ng / $\mathrm{m}^{3} \mathrm{no}$ ar, 4,1 $\mu \mathrm{g} / \mathrm{g}$ em deposição seca e $48 \mathrm{\mu g} / \mathrm{g}$ de lipídio em folhas. Estes autores afirmam que as folhas das plantas são eficazes na captação de uma variedade de PFAS transportados pelo ar, incluindo diésteres de ácido fosfórico polifluoroalquílico, capazes de atuar como uma abordagem de amostragem de ar passivo para monitoramento do ar.

O cultivo de culturas energéticas em aterros representa um desafio importante para o futuro próximo, uma vez que há possibilidade de utilizar locais desvalorizados para a produção de energia é muito atraente, Pivato et al. (2018) A diminuição da produção de chorume devido à presença de culturas energéticas na cobertura superior, o que aumenta a evapotranspiração, representa um aspecto favorável.

\section{CONCLUSÕES}

As pesquisas em relação a disposição dos resíduos e seus impactos são crescentes, contudo ainda são limitadas as áreas impactadas, constatou-se que as poluições ocasionadas pelos resíduos sólidos ultrapassam as barreiras territoriais, contaminando pessoas de outros locais. Há necessidade de ampliar as pesquisas, pois os impactos não são apenas ambientais afeta diretamente a qualidade de vidas das pessoas 
e dos animais. A gestão dos resíduos sólidos para forma adequada de disposição dos resíduos é fundamental para desenvolvimento sustentável populacional.

\section{REFERÊNCIAS}

ADEMOLA, A. K.; AYO, I.; FOLASADE, O.; ONYINQUE, D.; EMMANUEL, E.. Assessments of natural radioactivity and determination of heavy metals in soil around industrial dumpsites in Sango-Ota, Ogun state, Nigeria. Journal of Medical Physics, v.39, n.2, p.106-111, 2014. DOI: http://doi.org/10.4103/0971-6203.131285

AKORTIA, E.; OLUKUNLE, O. I.; DASO, A. P.; OKONKWO, J. O.. Soil concentrations of polybrominated diphenyl ethers and trace metals from an electronic waste dump site in the Greater Accra Region, Ghana: Implications for human exposure. Ecotoxicol Environ Saf, v.137, n.1, p.247-255, 2017. DOI: http://doi.org/10.1016/j.ecoenv.2016.12.008

AL-DELAIMY, W. K.; LARSEN, C. W.; PEXXOLI, K.. Differences in health symptoms among residents living near illegal dump sites in Los Laureles Canyon, Tijuana, Mexico: a cross sectional survey. Int J Environ Res Public Health, v.11, n.9, p.9532-9552, 2014. DOI:

http://doi.org/10.3390/ijerph110909532

AL-KHATIB, I. A.; HAMMAD, A. A.; SHARKAS, O. A.; SATO, C.. Public concerns about and perceptions of solid waste dump sites and selection of sanitary landfill sites in the West Bank, Palestinian territory. Environmental Monitoring and Assessment, v.187, n.4, p.186, 2015.

ALVES, J. B.; MENDONÇA, F. A.; DAIBEM, R. P.. Resíduos sólidos na área urbana de Fazenda Rio Grande/PR: a produção socioambiental do espaço urbano na cidade periférica. 0 espaço geográfico em análise, v.44, p.124-138, 2018. DOI: http://doi.org/10.5380/raega

BRASIL. Lei № 12.305: Institui a Política Nacional de Resíduos Sólidos; altera a Lei no 9.605, de 12 de fevereiro de 1998; e dá outras providências. Brasília: Diário Oficial União, 2010.

CHAKRABORTY, P.; SELVARAJ, S.; NAKAMURA, M.; PRITHIVIRAJ, B.; CINCINELLI, A.; BANG, J. J.. PCBs and $\mathrm{PCDD} / \mathrm{Fs}$ in soil from informal e-waste recycling sites and open dumpsites in India: Levels, congener profiles and health risk assessment. Science of the Total Environment, v.621, n.1, p.930-938, 2018. DOI:

http://doi.org/10.1016/j.scitotenv.2017.11.083

CHUNG, S. S.; ZHENG, J. S.; BURKET, S. R.; BROOKS, B. W.. Select antibiotics in leachate from closed and active landfills exceed thresholds for antibiotic resistance development. Environment International, v.115, n.1, p.89-96, 2018. DOI: http://doi.org/10.1016/j.envint.2018.03.014

DANTAS NETO, J.; SOUSA, L. A.. Parâmetros utilizados em levantamentos fitossociológicos para a recuperação de áreas degradadas: principais técnicas utilizadas em estudos recentes no Brasil. In: ARAUJO, S. M. S.; DANTAS NETO, J.. Recuperação de áreas degradadas: conceito, temas e casos. Curitiba, 2016. p.83-100.
FREDENSLUND, A. M.; MONSTER, J.; KJELDSEN, P.; SCHEUTZ, C.. Development and implementation of a screening method to categorise the greenhouse gas mitigation potential of 91 landfills. Waste Management, 2018. DOI: http://doi.org/10.1016/j.wasman.2018.03.005

GALVÃO, T. F.; PEREIRA, M. G.. Revisões sistemáticas da literatura: passos para sua elaboração. Epidemiologia Serviços Saúde, v.23, n.1, p.183-184, 2014.

GALVÃO, T. F.; PEREIRA, M. G.. Revisões sistemáticas da literatura: passos para sua elaboração. Epidemiol. Serv. Saúde, v.23, n.1, p.183-184, 2014. DOI: http://doi.org/10.5123/S1679-4974201400010001

JACOBI, P. R.. Desafios e reflexões sobre resíduos sólidos nas cidades brasileiras. In: SANTOS L, M. C.; DIAS, S. L. F. G.. Resíduos sólidos urbanos e seus impactos socioambientais. São Paulo: IEE-USP, 2012. p.82.

KHALIL, C.; HAGEH, C. A.; KORFALI, S.; KHNAYZER, R. S.. Municipal leachates health risks: Chemical and cytotoxicity assessment from regulated and unregulated municipal dumpsites in Lebanon. Chemosphere, v. 208, n.1, p. 1-13, 2018.

LIMA, A. P.; SANTOS, C. N.; CARVALHO, H. G.; FERNANDES, J. A. T.; UMIJI, S. S.; URYU, T. M. F.. Política Nacional de Resíduos Sólidos: eficácia da lei na cidade de Mogi das Cruzes. Revista Diálogos Interdisciplinares, v.7, n.2, p.24-39, 2018.

LIU, Q.; LI, Q.; WANG, N.; LIU, D.; ZAN, L.; CHANG, L.; GOU, $\mathrm{X}$.; WANG, P.. Bioremediation of petroleum-contaminated soil using aged refuse from landfills. Waste Management, 2018. DOI: http://doi.org/10.1016/i.wasman.2018.05.010

LIU, Y.; LU, W.; WANG, H.; GAO, X.. Odor impact assessment of trace sulfur compounds from working faces of landfills in Beijing, China. Journal Environmental Management, v.220, n.1, p.136-141, 2018. DOI:

http://doi.org/10.1016/j.jenvman.2018.04.122

NGWABIE, N. M.; WIRLEN, Y. L.; YINDA, G. S.; VANDERZAAG, A. C.. Quantifying greenhouse gas emissions from municipal solid waste dumpsites in Cameroon. Waste Management, 2018. DOI: http://doi.org/10.1016/j.wasman.2018.02.048

O'SHEA, F. T.; CUNDY, A. B.; SPENCER, K. L.. The contaminant legacy from historic coastal landfills and their potential as sources of diffuse pollution. Marine Pollution Bulletin, v.128, n.1, p.446-455, 2018. DOI:

http://doi.org/10.1016/j.marpolbul.2017.12.047

PATEL, M. L.; JAIN, R.; SAXENA, A.. Assessment of the Municipal Solid Waste \& Status of Implementation of Municipal Solid Vaste (Management \& Mandinga), Rufles, 2000 in Ihe Skate off Maya Predes, 2008 - A case stud. Vaste Management \& Research, v.29, p.558-562, 2010. DOI: http://doi.org/10.1177/0734242X10372662 
PIVATO, A.; LAVAGNOLO, M.C.; RAGA, R.; GARBO, F.; MORETTO, M.; COSSU, R.. Energy crops on landfills: functional, environmental, and costs analysis of different landfill configurations. Environmental Science Pollution Research, v.1, n.1, p.1-13, 2018. DOI: http://doi.org/10.1007/s11356-018-1452-1

SAMPAIO, R. F.; MANCINI, M. C.. Estudos de revisão sistemática: um guia para síntese criteriosa da evidência cientifica. Revista brasileira de fisioterapia, v.11, n.1, p.8389, 2007.

SÁNCHEZ, A. M. S.; SANCHEZ, F. J. S.; RUIZ-MUÑOZ, D.. Riesgos laborales en las empresas de residuos sólidos em Andalúcia: uma perspectiva de género. Saúde e Sociedade, v.25, n.3, p.798-810, 2017. DOI: http://doi.org/10.1590/S0104-12902017162878

STEFANIA, G. A.; ZANOTTI, C.; BONOMI, T.; FUMAGALLI, L.; ROTIROTI, M.. Determination of trigger levels for groundwater quality in landfills located in historically human-impacted areas. Waste Management, v.75, n.1, p.400-406, 2018. DOI:

http://doi.org/10.1016/j.wasman.2018.01.043

TAULER-AMETLLER, H.; HERNANDEZ-MATIAS, A.; PÁRES, F.; REAL, J.. Assessing the applicability of stable isotope analysis to determine the contribution of landfills to vultures' diet. PLoS One, v.13, n.5, 2018. DOI: http://doi.org/10.1371/journal.pone.0196044
TIAN, Y.; YAO, Y.; CHANG, S.; ZHAO, Z.; ZHAO, Y.; YUAN, X.; WU, F.; SUN, H.. Occurrence and Phase Distribution of Neutral and lonizable Per- and Polyfluoroalkyl Substances (PFASs) in the Atmosphere and Plant Leaves around Landfills: A Case Study in Tianjin, China. Environmental Science \& Technology, v.52, n.3, p.1301-1310, 2018. DOI: http://doi.org/10.1021/acs.est.7b05385

TONGESAYI, T.; KUGARA, J.; TONGESAYI, S.. Waste dumpsites and public health: a case for lead exposure in Zimbabwe and potential global implications. Environmental Geochemistry and Health, v.40, n.1, p.375-381, 2018.

WALDMAN, M.. Lixo: cenários e desafios. São Paulo: Cortez, 2010. p.231.

WANG, Y.; LI, L.; KUBOTA, J.; HAN, R.; ZHU, X.; LU, G.. Does urbanization lead to more carbon emission?. Evidence from a panel off BRICS countries. Applied Energy, v.168, n.1, p.375-380, 2016

YOU, X.; WU, D.; WEI, H.; XIE, B.; LU, J.. Fluoroquinolones and beta-lactam antibiotics and antibiotic resistance genes in autumn leachates of seven major municipal solid waste landfills in China. Environment International, v.113, n.1, p.162-169, 2018. DOI: http://doi.org/10.1016/j.envint.2018.02.002 